\title{
Efficacy of Roflumilast in Bronchiectasis Patients with Frequent Exacerbations: A Double-Blinded, Randomized, Placebo- Controlled Pilot Clinical Trial
}

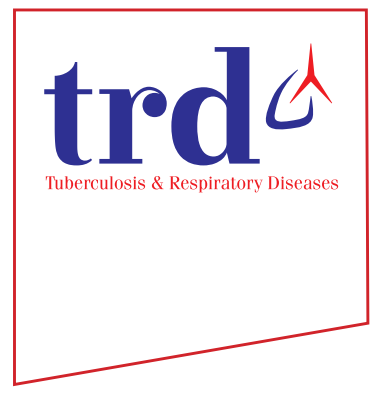

\author{
Siwasak Juthong, M.D. (ii) and Pattaraporn Panyarath, M.D. \\ Department of Internal Medicine, Faculty of Medicine, Prince of Songkla University, Hat Yai, Thailand
}

Background: Bronchiectasis patients with neutrophilic airway inflammation develop symptoms of chronic cough, sputum production, and recurrent exacerbations. Roflumilast has anti-inflammatory actions via decreased neutrophilic airway inflammation. The effectiveness of roflumilast to reduce bronchiectasis exacerbation has never been evaluated. Methods: We conducted a double-blinded, randomized, placebo-controlled trial. Our primary objective was to assess the effect of roflumilast compared with that of a placebo in reducing exacerbation rates in bronchiectasis patients. The secondary objectives were the changes in forced expiratory volume in 1 second $\left(\mathrm{FEV}_{1}\right)$ and St. George's Respiratory Questionnaire (SGRQ). Bronchiectasis patients older than 18 years who had had two exacerbations during the previous 12 months were randomly assigned to receive either $500 \mu \mathrm{g}$ of either roflumilast or a placebo once daily for 6 months in a 1:1 ratio.

Results: Forty bronchiectasis patients who had experienced exacerbations were screened. Thirty patients completed the study after 6 months of treatment: roflumilast group $(n=15)$ and placebo group $(n=15)$. The rates of exacerbations were 0.57 and 0.59 per patient in the roflumilast and placebo groups, respectively. Prebronchodilator $\mathrm{FEV}_{1}$ increased by $0.07 \mathrm{~L}$ from baseline in the roflumilast group and decreased by $0.015 \mathrm{~L}$ in the placebo group, but the difference was not significant. No significant differences were observed in the change of SGRQ scores between the roflumilast and placebo groups. Roflumilast had significant side effects, including loss of appetite and headache.

Conclusion: Roflumilast did not significantly affect the rate of exacerbations or quality of life. However, $\mathrm{FEV}_{1}$ tended to improve more in the roflumilast group than in the placebo group.

Keywords: Bronchiectasis; Exacerbations; Lung functions; Roflumilast

Address for correspondence: Siwasak Juthong, M.D.

Department of Internal Medicine, Faculty of Medicine, Prince of Songkla University, Hat Yai, Songkhla 901 10, Thailand

Phone: 6674-451-474, Fax: 6674-455-855, E-mail: jsiwasak@medicine.psu.ac.th

Address for co-correspondence: Pattaraporn Panyarath, M.D.

Department of Internal Medicine, Faculty of Medicine, Prince of Songkla University, Hat Yai, Songkhla 90110, Thailand

Phone: 6674-45-1474, Fax:6674-451494, E-mail: zealot_zine@hotmail.com

Received: Mar. 23, 2021, Revised: May. 24, 2021, Accepted: Aug. 1, 2021, Published online: Aug. 3, 2021

(a) It is identical to the Creative Commons Attribution Non-Commercial License (http://creativecommons.org/licenses/by-nc/4.0/). 


\section{Introduction}

Bronchiectasis patients have clinical symptoms of chronic cough, productive sputum, hemoptysis, recurrent pulmonary exacerbations, and frequent hospitalization, resulting in dyspnea, low quality of life, and high mortality ${ }^{1}$. Bronchiectasis is a chronic neutrophilic airway inflammation ${ }^{2}$. Neutrophils are key components of lung damage and dilated bronchi ${ }^{3,4}$. Bronchiectasis is characterized by a vicious cycle of airway inflammation, poor mucus clearance, bacterial colonization, airway obstruction, and progressive tissue destruction ${ }^{1}$. The strategy is to break the vicious cycle to improve patient outcomes. The goals of therapeutic approaches are management of chronic bacterial infections and prevention of exacerbations ${ }^{1}$.

Pulmonary exacerbations of bronchiectasis are defined by an increase in daily respiratory symptoms, such as cough, sputum production, breathlessness, malaise, and fatigue ${ }^{5}$. Bronchiectasis patients with frequent exacerbations have a low quality of life and increased mortality ${ }^{6}$. The European Respiratory Society ${ }^{7}$ and British Thoracic Society bronchiectasis guidelines emphasize exacerbation prevention and improvement in the symptoms and quality of life of the patients ${ }^{1}$.

The chronic bronchitis component of bronchiectasis is a shared common mechanism in chronic obstructive pulmonary disease (COPD). Roflumilast is an anti-inflammatory agent and inhibitor of phosphodiesterase 4 that significantly reduces exacerbations in COPD with chronic bronchitis and a high baseline frequency of exacerbations ${ }^{8}$. The anti-inflammatory actions of roflumilast are to increase the cyclic adenosine monophosphate level and decrease the neutrophil-driven airway inflammation, reduce neutrophil chemotaxis, decrease release of pro-inflammatory mediators, and induce neutrophil apoptosis ${ }^{9}$. Therefore, it is logical to consider roflumilast for the treatment of bronchiectasis.

Park $^{10}$ reported a phase II trial using roflumilast $250 \mu \mathrm{g}$ or $500 \mu \mathrm{g}$ for a 16 -week duration in nine symptomatic bronchiectasis patients. The participants had improved health-related quality of life measured by the COPD assessment test score and the St. George's Respiratory Questionnaire (SGRQ), but the results did not achieve statistical significance. The study did not report on exacerbations or lung-function tests, which would be the most important clinical trial end-points for bronchiectasis patients ${ }^{11}$. To date, no study has reported on using roflumilast to reduce exacerbations or lung-function tests in bronchiectasis patients with frequent exacerbations. We did a study to assess the effect of roflumilast on the exacerbation of bronchiectasis.

\section{Materials and Methods}

\section{Study design and patients}

We conducted a prospective preliminary double-blinded, randomized, placebo-controlled trial in Songklanagarind Hospital, Thailand, from January 2015 to November 2015. The participants included: (1) bronchiectasis patients older than 18 years at the outpatient department and diagnosed as having bronchiectasis by high-resolution computed tomography (HRCT) and (2) bronchiectasis patients who had had a history of exacerbation equal to or more than 2 times in the previous 12 months. We defined the exacerbation of bronchiectasis as being that of patients who had had at least three of these symptoms: increased cough, sputum production, change in sputum color, breathlessness, or hemoptysis; and for whom the doctor had prescribed antibiotics. The exclusion criteria were for patients: ( 1 ) diagnosed as COPD, with active tuberculosis, interstitial lung disease, or lung cancer; (2) with cirrhosis or psychiatric disease; (3) with a smoking history of more than 15 pack-years; (4) with previous use of systemic antibiotics or theophylline therapy within 30 days before study entry; (5) in lactation period; (6) with a body weight less than $30 \mathrm{~kg}$; or (7) who had contraindications for doing spirometry or the 6-minute walk test (6MWT).

This study was approved by the Office of Human Research Ethics Committee at the Faculty of Medicine, Prince of Songkla University, Thailand. The Ethics Committee approval number was EC 57-261-14-4, dated 23 February 2015. This study was supported by institutional funding. We followed the CONSORT 2010 statement from www.consort-statement.org.

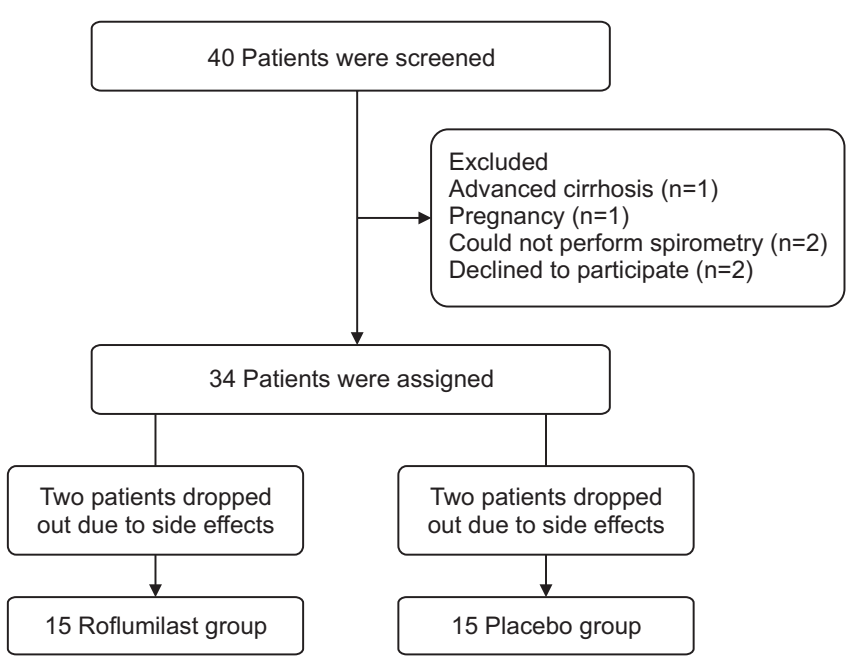

Figure 1. Patient flow diagram. 


\section{Study objectives}

Our primary objective in this study was to compare exacerbation rates in bronchiectasis patients who had received roflumilast or who had received a placebo during a 6-month follow-up period. The secondary objectives were to describe patient-reported outcomes between patients who had re- ceived roflumilast or who had received a placebo based on the results of forced expiratory volume in 1 second $\left(\mathrm{FEV}_{1}\right)$, SGRQ, and the 6MWT.

\section{Sample size}

This study was a pilot phase III double-blinded, random-

\section{Table 1. Baseline characteristics}

\begin{tabular}{|c|c|c|c|}
\hline & Roflumilast $(\mathrm{n}=15)$ & Placebo $(n=15)$ & p-value (95\% CI) \\
\hline Age (mean $\pm S D), y r$ & $56.6 \pm 10.4$ & $57.0 \pm 9.3$ & 0.840 \\
\hline Men & $6(40.0)$ & $2(13.3)$ & 0.100 \\
\hline Smoking status & & & 0.042 \\
\hline Never smoking & $10(66.7)$ & $15(100)$ & \\
\hline Current smoker & $1(6.7)$ & $0(0)$ & \\
\hline History smoker & $4(26.7)$ & $0(0)$ & \\
\hline Smoking history, pack-years & $1.40 \pm 3.54$ & 0 & \\
\hline Body mass index, $\mathrm{kg} / \mathrm{m}^{2}$ & 20.78 & 21.48 & \\
\hline Weight, kg & $51.88 \pm 7.03$ & $50.95 \pm 12.31$ & $0.800(-6.5$ to 8.4$)$ \\
\hline Height, cm & $158.60 \pm 8.86$ & $154.26 \pm 8.48$ & $0.180(-2.15$ to 10.8$)$ \\
\hline Exacerbations in past year & $4.20 \pm 2.85$ & $3.00 \pm 2.29$ & $0.210(-0.74$ to 3.14$)$ \\
\hline \multicolumn{4}{|l|}{ Spirometry before bronchodilation } \\
\hline $\mathrm{FEV}_{1}, \mathrm{~L}$ & $1.48 \pm 0.54$ & $1.15 \pm 0.41$ & $0.070(-0.37$ to 0.68$)$ \\
\hline FVC, L & $2.16 \pm 0.60$ & $1.75 \pm 0.55$ & $0.060(-0.2$ to 0.84$)$ \\
\hline $\mathrm{FEV}_{1} / \mathrm{FVC}, \%$ & $67.73 \pm 13.44$ & $66.46 \pm 11.47$ & $0.780(-8.08$ to 10.61$)$ \\
\hline Total scores on St. George's Respiratory Questionnaire & $48.99 \pm 16.04$ & $33.73 \pm 20.01$ & $0.020(1.6$ to 28.8$)$ \\
\hline Symptoms & $53.83 \pm 24.98$ & $43.57 \pm 24.38$ & $0.260(-8.20$ to 28.2$)$ \\
\hline Activity & $54.20 \pm 21.6$ & $41.25 \pm 25.64$ & $0.140(-4.79$ to 30.6$)$ \\
\hline Impacts & $44.51 \pm 18.08$ & $26.75 \pm 21.80$ & $0.020(2.7$ to 32.7$)$ \\
\hline 6-Minute walk test distance $($ mean $\pm S D), m$ & $422.60 \pm 67.13$ & $410.06 \pm 41.30$ & $0.560(-29.6$ to 53.69$)$ \\
\hline Respiratory drugs & & & $>0.999$ \\
\hline Any & $5(33.3)$ & $6(40.0)$ & \\
\hline SABAs & $2(13.3)$ & $2(13.3)$ & \\
\hline LABAs & $2(13.3)$ & $1(6.7)$ & \\
\hline Corticosteroid inhaled with LABA & $2(13.3)$ & $1(6.7)$ & \\
\hline \multicolumn{4}{|l|}{ Vaccination history in past year } \\
\hline Influenza vaccine & $11(73.3)$ & $11(73.3)$ & $>0.999$ \\
\hline Pneumococcal vaccine & $8(53.3)$ & $6(40.0)$ & 0.71 \\
\hline Pulmonary rehabilitation mMRC dyspnea scale & $0(0)$ & $2(13.3)$ & 0.48 \\
\hline 1 & $1(6.7)$ & $5(33.3)$ & 0.16 \\
\hline 2 & $13(86.7)$ & $10(66.7)$ & \\
\hline 3 & $1(6.7)$ & $0(0)$ & \\
\hline
\end{tabular}

Values are presented as mean \pm SD or number (\%) unless otherwise indicated.

$\mathrm{CI}$ : confidence interval; SD: standard deviation; $\mathrm{FEV}_{1}$ : forced expiratory volume in 1 second; FVC: forced vital capacity; $\mathrm{SABA}$ : short acting $\beta 2$ agonist; LABA: long-acting $\beta 2$ agonist; mMRC: modified Medical Research Council. 
ized, placebo-controlled clinical trial. Forty bronchiectasis patients were screened, and 15 patients were randomized into each group (total sample=30).

\section{Study drugs and measurements}

Roflumilast (Daxas; Takeda, Osaka, Japan) 500- $\mu$ g tablets were manufactured by Takeda Pharmaceutical Company. Placebo tablets were manufactured by the Department of Pharmaceutical Manufacturing, Faculty of Pharmaceutical Sciences, Prince of Songkla University, Thailand. All patients included in the study met the inclusion criteria and signed informed consent forms. They were then computer randomized 1:1 with a block of four to receive once daily $500 \mu \mathrm{g}$ of either roflumilast or a placebo for 24 weeks. All patient baseline characteristics were recorded, including smoking status, current and past chest symptoms, possible etiology of bronchiectasis, concurrent respiratory medications, history of vaccinations, pulmonary rehabilitation, and history of bronchiectasis exacerbations in the previous 12 months. Data recorded at the first visit and at the 24-week visit at the end of the study protocol included the SGRQ, pulmonary function tests (i.e., $\mathrm{FEV}_{1}$, forced vital capacity [FVC]), 6MWT, and diffusing capacity for carbon monoxide.

\section{Study protocol}

All participants signed the informed consent forms, and all patients continued to use the previous baseline standard treatments. We then randomized them to receive once daily $500 \mu \mathrm{g}$ of either roflumilast or the placebo for 24 weeks. The patients had scheduled visits every 4 weeks for the surveillance of acute exacerbation and the potential for adverse drug effects. If the patients had an exacerbation of bronchiectasis, they were treated with the appropriate antibiotic for 10 days. We did sputum cultures for aerobic bacteria at the first visit and at the end of the study protocol. All participants were informed of pulmonary rehabilitation (e.g., breathing exercises and postural drainage), and they continued to use prior medications.

\section{Statistical analysis}

Since our clinical trial was a pilot study, we did not do a sample-size calculation. Therefore, the size of the samples possibly affected the statistical significance of this study. We used the Stata version 14 program for the analysis. Continuous variables (demographic data) are presented as mean \pm standard deviation and were compared using the Student t-test. Noncontinuous variables are presented as numbers and percentages, which we compared using the chi-squared and Fisher exact tests. We used a Kaplan-Meier estimation curve and Cox's proportional hazards model to identify the first exacer- bation, and used the Poisson regression model to assess the rate of event-based exacerbations per patient. A two-tailed $\mathrm{p}<0.05$ was considered statistically significant. We analyzed the outcomes as per protocol analysis.

\section{Results}

\section{Demographics of patients}

Forty bronchiectasis patients confirmed by HRCT were screened. Initially, the patients were randomized into the treatment group $(\mathrm{n}=17)$ and control group $(\mathrm{n}=17)$. However, 15 patients in each group completed the study. Two patients in the roflumilast group and two in the placebo group dropped out at week 1 because of adverse-effect intolerance. Finally, 15 patients each in the treatment and control groups continued until the end of the study (Figure 1). Demographic and clinical characteristics at the time of study entry are presented in Table 1. The median ages were $56.6 \pm 10.4$ and $57.0 \pm 9.3$ years for the bronchiectasis group and placebo group, respectively. Twenty-two patients $(73.3 \%)$ were women, whose mean age was 57 years. The mean number of exacerbations in the previous year before study entry were $4.2 \pm 2.85$ and $3.1 \pm 1.95$ times for the bronchiectasis and placebo groups, respectively $(\mathrm{p}=0.21)$. There were no differences in the baseline characteristics between the two groups, such as body mass index, $\mathrm{FEV}_{1}$, $\mathrm{FVC}, \mathrm{FEV}_{1} / \mathrm{FVC}$, history of previous respiratory drug use, vaccinations, 6MWT, or modified Medical Research Council dyspnea scale (Table 1). At baseline, the roflumilast group had total SGRQ scores higher than those of the placebo group (49.0 points vs. 33.7 points) $(\mathrm{p}=0.02)$.

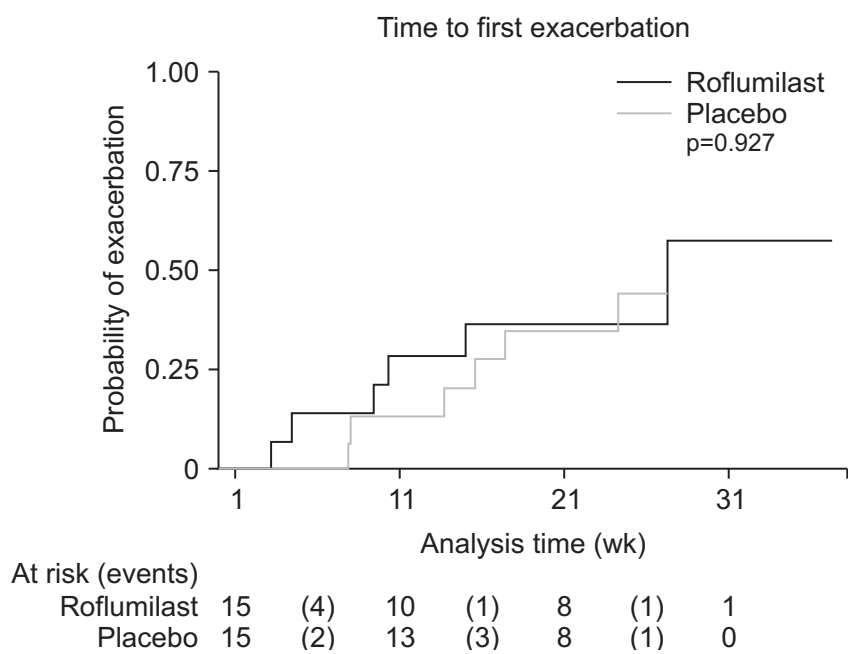

Figure 2. Kaplan-Meier curve for the time of first exacerbation in bronchiectasis patients who received roflumilast or placebo. 


\section{Exacerbations}

After the completion of the 6-month study, the numbers of exacerbations in the roflumilast and placebo groups were 12 and 13 times, respectively. The rates of event-based exacerbations in the roflumilast and placebo groups were 0.57 and 0.59 times/patient, respectively, which was not significantly different. The rate ratio was 0.97 (95\% confidence interval [CI], $0.28-3.04 ; \mathrm{p}=0.96$ ). The lengths of time to the first exacerbation were 9.3 weeks in the patients who received roflumilast and 10.1 weeks in the placebo group ( $\mathrm{p}=0.92$ ) (Figure 2).

\section{Pulmonary function tests}

After 6 months of roflumilast, the prebronchodilator $\mathrm{FEV}_{1}$ increased $70 \mathrm{~mL}$ compared with a decrease of $15 \mathrm{~mL}$ in the placebo group. but this was not a significant difference (difference $=85 \mathrm{~mL} ; 95 \% \mathrm{CI},-0.08$ to $0.25 ; \mathrm{p}=0.28$ ). The prebronchodilator FVC decreased $10 \mathrm{~mL}$ in the roflumilast group and decreased $1 \mathrm{~mL}$ in the placebo group (difference $=9 \mathrm{~mL} ; 95 \%$
$\mathrm{CI},-0.19$ to $0.16 ; \mathrm{p}=0.88$ ). The $6 \mathrm{MWT}$ increased $21.0 \mathrm{~m}$ in the roflumilast group and $22.8 \mathrm{~m}$ in the placebo group (difference $=1.8 \mathrm{~m} ; 95 \% \mathrm{CI},-66.9$ to $63.2 ; \mathrm{p}=0.95$ ) (Table 2).

\section{Health-related quality of life}

Following the 6-month study period, the total scores of the SGRQ decreased 13.5 and 5.5 points in the roflumilast and placebo groups, respectively (difference $=8$ points; $95 \%$ CI, -20.3 to $4.4 ; \mathrm{p}=0.19$ ). The SGRQ symptoms, activity, and impacts scores were not statistically significantly different between the roflumilast and placebo groups (Table 2).

\section{Adverse events}

There were significantly more bronchiectasis patients in the roflumilast group than in the placebo group who had adverse effects ( 9 vs. 2 patients, $\mathrm{p}=0.02$ ). The most common side effects of roflumilast were loss of appetite or fatigue (46.7\%), headache $(33.3 \%)$, nausea or vomiting $(26.7 \%)$, and diarrhea

Table 2. Patient-reported outcomes and changes from baseline at 6 months after initiation of treatment

\begin{tabular}{|c|c|c|c|c|}
\hline & \multicolumn{2}{|c|}{ Change from baseline } & \multirow[b]{2}{*}{ Difference (95\% CI) } & \multirow[b]{2}{*}{ p-value } \\
\hline & $\begin{array}{c}\text { Roflumilast group } \\
(n=15)\end{array}$ & $\begin{array}{c}\text { Placebo group } \\
\quad(n=15)\end{array}$ & & \\
\hline Prebronchodilator $\mathrm{FEV}_{1}, \mathrm{~L}$ & 0.07 & -0.01 & -0.08 to 0.25 & 0.280 \\
\hline Prebronchodilator FVC, L & -0.01 & -0.01 & -0.19 to 0.16 & 0.880 \\
\hline Prebronchodilator $\mathrm{FEV}_{1} / \mathrm{FVC}, \%$ & 1.40 & -1.14 & -0.12 to 5.20 & 0.060 \\
\hline Total score on St George's Respiratory Questionnaire & -13.46 & -5.54 & -20.27 to 4.41 & 0.190 \\
\hline Symptoms & -19.66 & -8.23 & -27.47 to 4.60 & 0.150 \\
\hline Activity & -8.19 & -6.05 & -20.25 to 15.96 & 0.800 \\
\hline Impacts & -16.52 & -3.85 & -27.65 to 2.32 & 0.090 \\
\hline 6-Minute walk test distance, $\mathrm{m}$ & 21.00 & 22.80 & -66.93 to 63.22 & 0.950 \\
\hline
\end{tabular}

CI: confidence interval; $\mathrm{FEV}_{1}$ : forced expiratory volume in 1 second; $\mathrm{FVC}$ : forced vital capacity.

Table 3. Adverse events from roflumilast and placebo

\begin{tabular}{|lccc|}
\hline & Roflumilast $(\mathbf{n}=\mathbf{1 5})$ & Placebo $(\mathbf{n}=\mathbf{1 5})$ & p-value (95\% CI) \\
\hline Any adverse events & $9(60.0)$ & $2(13.3)$ & 0.020 \\
\hline Adverse events & & & \\
\hline Diarrhea & $2(13.3)$ & $1(6.7)$ & 0.500 \\
\hline Loss of appetite & $7(46.7)$ & 0 & 0.006 \\
\hline Nausea or vomiting & $4(26.7)$ & 0 & 0.050 \\
\hline Headache & $5(33.3)$ & $1(6.7)$ & 0.080 \\
\hline Body weight loss $(\mathrm{kg})$ & $-1.37 \pm 2.7$ & $-0.77 \pm 3.6$ & $0.610(-3.02$ to 1.82$)$ \\
\hline Compliance $>80.0 \%$ of total dose & $10(66.7)$ & $14(93.3)$ & 0.060 \\
\hline
\end{tabular}

Values are presented as number (\%) unless otherwise indicated.

CI: confidence interval. 
(13.3\%) (Table 3). The severities of the adverse events were grade 1 and grade 2 . Four patients quit the study drug or placebo: 2 in the roflumilast group because of nausea and two patients in the placebo group because of headache. Body weight losses in the roflumilast and placebo groups were $1.37 \mathrm{~kg}$ and $0.77 \mathrm{~kg}$, respectively, but did not reach statistical significance $(\mathrm{p}=0.61)$.

We assessed adherence to the protocol and found that the patients in the roflumilast and placebo groups who regularly used $80 \%$ of the total dose were 10 patients $(66.7 \%)$ and 14 patients $(93.3 \%)$, respectively $(\mathrm{p}=0.06)$.

\section{Discussion}

In the bronchiectasis patients who had experienced frequent exacerbations, treatment with $500 \mu \mathrm{g}$ of roflumilast once daily for 6 months did not significantly reduce exacerbations more than the placebo did. Roflumilast tended to improve $\mathrm{FEV}_{1}$ but this was not statistically significant compared with that produced by the placebo. The health-related quality of life and exercise capacity as assessed by the 6MWT were not significantly different between the two groups. The number of bronchiectasis patients who received roflumilast and had side effects, such as loss of appetite, fatigue, headache, nausea, vomiting, or diarrhea, was significantly greater.

Roflumilast has anti-neutrophilic inflammatory effects and significantly decreases exacerbations in frequent exacerbators with the chronic bronchitis phenotype of $\mathrm{COPD}^{8}$. In this study, roflumilast did not significantly reduce the time to the first exacerbation or the event rate of exacerbation in bronchiectasis. Bronchiectasis has a more complicated pathophysiology in exacerbation, such as more bacterial colonization, structural abnormality, and abnormal mucus clearance, than does COPD. Roflumilast inhibits inflammation but has no effect in reducing bacterial colonization or mucus clearance. Our pilot study had relatively few bronchiectasis patients. Therefore, a similar study may need a larger number of patients in order to find out how roflumilast affects the exacerbation rate. The side effects of roflumilast possibly affected adherence to the drug. The heterogeneity of bronchiectasis exacerbation is due to multiple mechanisms ${ }^{12}$, such as the infection or inflammatory process or both.

Roflumilast tended to improve $\mathrm{FEV}_{1}$ more than the placebo did. After 24 weeks of roflumilast therapy, the $\mathrm{FEV}_{1}$ increased $70 \mathrm{~mL}$ in bronchiectasis patients, which was similar to that of COPD patients, who had an improvement in $\mathrm{FEV}_{1}$ of $57 \mathrm{~mL}^{13}$. The mechanism of an increased $\mathrm{FEV}_{1}$ with roflumilast therapy is not fully understood. Roflumilast may inhibit neutrophilic airway inflammation ${ }^{14}$ and thus break the vicious cycle of infection and inflammation that affects the airway caliber. However, roflumilast has been reported to have no effect on airway smooth-muscle relaxation ${ }^{15}$. The structural change in bronchiectasis is considered to be irreversible; therefore, a drug that can increase $\mathrm{FEV}_{1}$ may be interesting.

In this study, roflumilast improved the quality of life as assessed by the SGRQ, with an 8-point difference after 6 months of therapy. Although the total scores of the SGRQ changed after treatment, they were not significantly different from those of the placebo group. However, the change met the minimal clinically important difference of 4 points in the total scores of the SGRQ. The improved quality of life by roflumilast was the same in as a study by Park ${ }^{10}$, in which roflumilast significantly improved the quality of life. In this study, roflumilast improved $\mathrm{FEV}_{1}$ but did not improve the exercise capacity, which would improve the symptoms of dyspnea and quality of life. The results of the 6-minute walk test distance showed no significant difference between the roflumilast and placebo groups, possibly because the patients were less dyspneic, as based on the scores of the modified Medical Research Council scale; these were mainly 1 and 2 in both groups.

In bronchiectasis patients, roflumilast had significantly more side effects than in the placebo group. These side effects were not serious adverse events and were not life threatening. The most common side effect of roflumilast in bronchiectasis was loss of appetite or fatigue, whereas diarrhea ${ }^{8}$ was most common in COPD patients. The roflumilast patients experienced a weight loss of $1.37 \mathrm{~kg}$ compared with $2.09 \mathrm{~kg}$ in a COPD study ${ }^{13}$. Weight loss can affect the quality of life because of a lower body mass index in bronchiectasis patients.

This study has some strengths and limitations. The first strength is the study design, which was the first randomized, double-blinded, placebo-controlled trial of roflumilast in bronchiectasis patients. Second, we selected bronchiectasis patients with frequent exacerbations who had a high clinical burden and severe disease. Third, roflumilast was taken for 6 months, which could demonstrate its effects on exacerbation and patient-reported outcomes. The limitations were that we had only a few bronchiectasis patients and conducted the study at a single center.

In bronchiectasis patients with frequent exacerbations, using $500 \mu \mathrm{g}$ of roflumilast daily for 6 months did not significantly affect the rate of exacerbations or quality of life more than the placebo did. However, it tended to improve lung functions and ameliorate the symptoms and impacts of bronchiectasis in a very small group of subjects in a short study. A longerterm prospective study with more bronchiectasis patients should be conducted.

\section{Authors' Contributions}

Conceptualization: Juthong S. Methodology: Juthong S. Formal analysis: Juthong S. Data curation: Panyarath P. Software: Juthong S, Panyarath P. Validation: Juthong S, Panyarath P. Investigation: Juthong S, Panyarath P. Writing - original draft 
preparation: Juthong S, Panyarath P. Writing - review and editing: Juthong S, Panyarath P. Approval of final manuscript: all authors.

\section{Conflicts of Interest}

No potential conflict of interest relevant to this article was reported.

\section{Acknowledgments}

The authors are grateful to Glenn Shingledecker for proofreading the English.

\section{Funding}

This study was supported by the Faculty of Medicine, Prince of Songkla University Research Fund.

\section{References}

1. Hill AT, Sullivan AL, Chalmers JD, De Soyza A, Elborn SJ, Floto AR, et al. British Thoracic Society Guideline for bronchiectasis in adults. Thorax 2019;74:1-69.

2. Chalmers JD, Moffitt KL, Suarez-Cuartin G, Sibila O, Finch S, Furrie E, et al. Neutrophil elastase activity is associated with exacerbations and lung function decline in bronchiectasis. Am J Respir Crit Care Med 2017;195:1384-93.

3. Dente FL, Bilotta M, Bartoli ML, Bacci E, Cianchetti S, Latorre M, et al. Neutrophilic bronchial inflammation correlates with clinical and functional findings in patients with noncystic fibrosis bronchiectasis. Mediators Inflamm 2015;2015:642503.

4. Chalmers JD, Aliberti S, Blasi F. Management of bronchiectasis in adults. Eur Respir J 2015;45:1446-62.

5. Hill AT, Haworth CS, Aliberti S, Barker A, Blasi F, Boersma W, et al. Pulmonary exacerbation in adults with bronchiectasis: a consensus definition for clinical research. Eur Respir J 2017;49:170051.
6. Chalmers JD, Aliberti S, Filonenko A, Shteinberg M, Goeminne PC, Hill AT, et al. Characterization of the "frequent exacerbator phenotype" in bronchiectasis. Am J Respir Crit Care Med 2018;197:1410-20.

7. Polverino E, Goeminne PC, McDonnell MJ, Aliberti S, Marshall SE, Loebinger MR, et al. European Respiratory Society guidelines for the management of adult bronchiectasis. Eur Respir J 2017;50:1700629.

8. Martinez FJ, Calverley PM, Goehring UM, Brose M, Fabbri LM, Rabe KF. Effect of roflumilast on exacerbations in patients with severe chronic obstructive pulmonary disease uncontrolled by combination therapy (REACT): a multicentre randomised controlled trial. Lancet 2015;385:857-66.

9. Sousa LP, Lopes F, Silva DM, Tavares LP, Vieira AT, Rezende $\mathrm{BM}$, et al. PDE4 inhibition drives resolution of neutrophilic inflammation by inducing apoptosis in a PKA-PI3K/Aktdependent and NF-kappaB-independent manner. J Leukoc Biol 2010;87:895-904.

10. Park J. Effect of roflumilast (Daxas) in patients with symptomatic non-cystic fibrosis bronchiectasis. Am J Respir Crit Care Med 2014;189:A6255.

11. Crichton ML, Aliberti S, Chalmers JD. A systematic review of pharmacotherapeutic clinical trial end-points for bronchiectasis in adults. Eur Respir Rev 2019;28:180108.

12. Chalmers JD, Boersma W, Lonergan M, Jayaram L, Crichton ML, Karalus N, et al. Long-term macrolide antibiotics for the treatment of bronchiectasis in adults: an individual participant data meta-analysis. Lancet Respir Med 2019;7:845-54.

13. Calverley PM, Rabe KF, Goehring UM, Kristiansen S, Fabbri LM, Martinez FJ, et al. Roflumilast in symptomatic chronic obstructive pulmonary disease: two randomised clinical trials. Lancet 2009;374:685-94.

14. Fabbri LM, Calverley PM, Izquierdo-Alonso JL, Bundschuh DS, Brose M, Martinez FJ, et al. Roflumilast in moderate-tosevere chronic obstructive pulmonary disease treated with longacting bronchodilators: two randomised clinical trials. Lancet 2009;374:695-703.

15. Bundschuh DS, Eltze M, Barsig J, Wollin L, Hatzelmann A, Beume R. In vivo efficacy in airway disease models of roflumilast, a novel orally active PDE4 inhibitor. J Pharmacol Exp Ther 2001;297:280-90. 